\title{
STRUCTURE THEORY OF FAITHFUL RINGS I. CLOSURE OPERATIONS ON LATTICES $\left({ }^{(1)}\right.$
}

\author{
BY \\ R. E. JOHNSON
}

A ring having no nonzero left annihilator will be called a faithful ring. Thus, if $R$ is a faithful ring, $a R \neq 0$ for every nonzero $a$ in $R$. This series of papers is concerned with the structure theory of faithful rings satisfying various types of minimum conditions.

The first paper of the series gives the lattice-theoretic background of our work. It has to do mainly with closure operations on a complete lattice $\mathscr{L}$ having the property that $\left(\bigcup_{i} A_{i}\right) \cap A=\bigcup_{i}\left(A_{i} \cap A\right)$ for every chain $\left\{A_{i}\right\} \subset \mathfrak{L}$ and $A \in \mathscr{L}$. The lattice of all (right) ideals of a ring has this property.

In the first section, the lattices $Q(\mathfrak{L})$ and $C(\mathscr{L})$ of all quasi-closure and closure operations respectively on $\mathscr{L}$ are defined and discussed. The second section deals with those subsets of $\mathscr{L}$, called insets, that are closed relative to the intersection operation and contain $I$. If $a \in C(\mathscr{L})$, the set $\mathfrak{L}^{a}$ of all $A \in \mathscr{L}$ such that $A^{a}=A$ is an inset of $\mathscr{L}$. The lattice $C(\mathscr{L})$ is shown to be dual isomorphic to the lattice $I(\mathscr{L})$ of all insets of $\mathscr{L}$ under the correspondence $a \rightarrow \mathscr{L}^{a}, a \in C(\mathscr{L})$. An $m$-closure operation on $\mathscr{L}$ is a closure operation that also is an $\cap$-endomorphism of $\mathscr{L}$. The set $C_{m}(\mathscr{L})$ of all $m$-closure operations on $\mathscr{L}$ is a complete sublattice of $C(\mathscr{L})$. If $\mathscr{L}$ is the lattice of all ideals of a ring $R$ and $\mathscr{L}^{\pi}$ is the inset of all semi-prime ideals of $R$, then $\pi$ is an example of an $m$ closure operation on $\mathcal{L}$.

Atomic closure operations on $\mathscr{L}$ are studied in the third section. Let $C^{0}(\mathscr{L})$ designate the subset of $C(\mathscr{L})$ containing all $a$ such that $0 \in \mathscr{L}^{a}$. The closure operation $a \in C^{0}(\mathscr{L})$ is atomic if $\mathscr{L}^{a}$ has minimal nonzero elements (atoms). If each nonzero $A \in \mathscr{L}^{a}$ contains an atom of $\mathscr{L}^{a}, a$ is called homogeneous. The set of all homogeneous $m$-closure operations on $\mathscr{L}$ is shown to be a dual ideal of $C_{m}^{0}(\mathcal{L})$.

The fourth section is concerned with closure operations on the direct product $\mathscr{L} \times \mathfrak{T}$ of the lattices $\mathscr{L}$ and $\mathfrak{T}$. Let $J(\mathscr{L} ; \mathfrak{T})(K(\mathscr{L} ; \mathfrak{T}))$ be the set of all $\cup$-homomorphisms $(\cap$-homomorphisms) of $\mathscr{L}$ into $\mathfrak{T}$ that map 0 into 0 ( $I$ into $I)$. If $x \in J(\mathscr{L} ; \mathfrak{T})$, the inverse mapping $x^{-1} \in K(\mathfrak{T} ; \mathfrak{L})$, and conversely. The algebraic systems $\{J(\mathscr{L} ; \mathfrak{T}) ; \leqq, \cup\}$ and $\{K(\mathfrak{T} ; \mathfrak{L}) ; \geqq, \cap\}$ are isomorphic under the correspondence $x \rightarrow x^{-1}$. Let $K^{\prime}(\mathfrak{T} ; \mathfrak{L})$ be the set of all $x \in K(\mathfrak{T} ; \mathfrak{L})$ such that $x\left(\bigcup_{i} M_{i}\right)=\bigcup_{i} x M_{i}$ for every chain $\left\{M_{i}\right\} \subset \mathscr{T}$. For $x \in K^{\prime}(\mathfrak{T} ; \mathfrak{L}), a \in C(\mathscr{L})$, and $b \in C(\mathfrak{T})$, necessary and sufficient conditions that $a x=x b$ are given. If $x \in K^{\prime}(\mathfrak{T} ; \mathfrak{L})$, the set of all $(a, b) \in C(\mathscr{L}) \times C(\mathfrak{T T})$

Presented to the Society, December 28, 1954; received by the editors June 6, 1956. tion.

(1) This research was supported in part by a grant from the National Science Founda- 
such that $a x=x b$ is a complete sublattice of $C(\mathscr{L}) \times C(\mathfrak{T})$.

Illustrations of the results of the previous sections are given in the fifth section with the right ideal lattice $\mathscr{L}$ of a faithful ring $R$. Again, these are concerned with the prime elements of $\mathscr{L}$.

The final section deals with modular lattices. If $c \in C_{m}^{0}(\mathfrak{L})$ and, for each $A \in \mathscr{L}^{c}, A \neq I, A \cap B=0$ for some nonzero $B \in \mathcal{L}$, then $c$ is said to be reducible. If $\mathscr{L}$ has a reducible $m$-closure operation $c$, then $c$ is unique. If $c$ is homogeneous, then both $\mathscr{L}^{c}$ and the center of $\mathscr{L}^{c}$ are atomic lattices [2, p. 130].

Subsequent papers of this series will contain applications of this paper to special types of faithful rings. The second paper is concerned with global properties of rings. Its principal results have to do with direct sum representations of modules and rings. These results generalize our previous ones on semi-prime rings [10]. The third paper will deal with local properties of rings. It will generalize our previous results on prime rings $[7 ; 8]$.

1. Closure operations. This paper is concerned solely with complete lattices $\{\mathfrak{L} ; \cup, \cap, \subset\}$ satisfying the following chain condition:

(C) For every chain $\left\{A_{i}\right\} \subset \mathscr{L}$ and $A \in \mathscr{L},\left(\cup_{i} A_{i}\right) \cap A=\bigcup_{i}\left(A_{i} \cap A\right)$.

Any complete lattice of subsets of a given set in which both the union operation on chains and the intersection operation are set-theoretic clearly satisfies (C). Thus, for example, this condition is satisfied by the lattice $\mathscr{L}$ of all (right) ideals of a ring. The identity elements of $\mathscr{L}$ relative to $U$ and $\cap$ are designated respectively by 0 and $I$ as usual.

A mapping $a: A \rightarrow A^{a}$ of a lattice $\mathcal{L}$ into itself is called a quasi-closure operation $[15$, p. 56] on $\&$ if and only if the following two conditions are satisfied: for every $A, B \in \mathcal{L}$, (C1) if $A \subset B$ then $A^{a} \subset B^{a}$; (C2) $A \subset A^{a}$. The set of all quasi-closure operations on $\mathfrak{L}$ will be designated by $Q(\mathfrak{L})$.

Operations $\cup$ and $\cap$ and a relation $\leqq$ may be defined on $Q(\mathscr{L})$ in an obvious way $\left[15\right.$, p. 55]. Thus, for $\left\{a_{i}\right\} \subset Q(\mathscr{L}), \cup_{i} a_{i}=b$ where $A^{b}=\bigcup_{i} A^{a_{i}}$; $\bigcap_{i} a_{i}=c$ where $A^{c}=\bigcap_{i} A^{a_{i}}, A \in \mathfrak{L}$. Also, for $a, b \in Q(\mathfrak{L})$,

$$
a \leqq b \text { if and only if } A^{a} \subset A^{b} \text { for every } A \in \mathcal{L} .
$$

It is evident that $\{Q(\mathscr{L}) ; \cup, \cap, \leqq\}$ is a complete lattice having $o: A^{\circ}=A$, $A \in \mathcal{L}$, and $i: A^{i}=I, A \in \mathcal{L}$, as its identity elements. If $\left\{a_{i}\right\}$ is a chain in $Q(\mathscr{L})$, then $\left\{A^{a_{i}}\right\}$ is a chain in $\mathscr{L}$. Thus it is clear that the chain condition (C) also holds for the lattice $Q(\mathscr{L})$.

The lattice $Q(\mathscr{L})$ also is closed under the iterative multiplication of mappings. Thus, for $a, b \in Q(\mathfrak{L}), A^{a b}=\left(A^{a}\right)^{b}, A \in \mathfrak{L}$. Some of the properties of this operation are listed below.

(P1) $a(b c)=(a b) c$.

(P2) $\quad a o=o a=a ; \quad a i=i a=i$.

(P3) $a\left(\cup_{i} a_{i}\right)=\cup_{i} a a_{i}$.

(P4) $a\left(\bigcap_{i} a_{i}\right)=\bigcap_{i} a a_{i}$.

(P5) $a \leqq b$ implies $a c \leqq b c$ and $c a \leqq c b$ for every $c \in Q(\mathfrak{L})$.

The multiplicative idempotent elements of $Q(\mathscr{L})$ are called closure operations on $\mathfrak{L}$. We shall designate by $C(\mathscr{L})$ the set of all closure operations on $\mathscr{L}$. 
If $\left\{a_{i}\right\} \subset C(\&)$ and $b=\bigcap_{i} a_{i}$, then $b \leqq a_{i}, b^{2} \leqq a_{i} b=\bigcap_{j} a_{i} a_{j} \leqq a_{i}^{2}=a_{i}$, and $b^{2} \leqq b$. We conclude that $C(\mathscr{L})$ is closed under the intersection operation. It is clear that $o, i \in C(\mathscr{L})$.

A closure operation * is defined on the lattice $Q(\mathscr{L})$ by $C(\mathscr{L})$. Thus, for each $a \in Q(\mathscr{L})$, let $a^{*}$ be the least element of $C(\mathscr{L})$ containing $a$. One may consider $a^{*}$ as the intersection of all $b \in Q(\mathcal{L})$ such that $b \geqq a$. Then ${ }^{*}: a \rightarrow a^{*}$, $a \in Q(\mathscr{L})$, is easily verified to be a closure operation on $Q(\mathscr{L})$. If we define the operation $\cup^{*}$ on $C(\mathfrak{L})$ as follows,

$$
\bigcup^{*}: \bigcup_{i}^{*} a_{i}=\left(\bigcup_{i} a_{i}\right)^{*}, \quad a_{i} \in C(\mathscr{L}),
$$

then evidently $\left\{C(\mathscr{L}) ; \cup^{*}, \cap, \leqq\right\}$ is a complete lattice.

The results given below on closure operations are similar to those of Hanai [5].

1.1. Lemma. For $a \in Q(\mathfrak{L})$ and $b \in C(\mathfrak{L}), a \leqq b$ if and only if $a b=b(b a=b)$.

Proof. If $a \leqq b, b=o b \leqq a b \leqq b^{2}$ and $a b=b$. Conversely, if $a b=b, a \leqq a b$ implies $a \leqq b$.

1.2. Theorem. For every $a, b \in C(\mathscr{L}), a \cup b \leqq a b \leqq a \cup^{*} b$.

Proof. Since $a \leqq a b$ and $b \leqq a b, a \cup b \leqq a b$. Also, $b \leqq a \cup^{*} b$ and therefore $a \bigcup^{*} b=\left(a \bigcup^{*} b\right) b$ by 1.1 . Hence $a b \leqq\left(a \cup^{*} b\right) b=a \bigcup^{*} b$, and 1.2 is proved.

A useful corollary of 1.2 is as follows.

1.3. Corollary. If $a, b$, and $a b$ are in $C(\mathcal{L})$, then $a b=b a=a \cup^{*} b$.

If $a b=b a$ for $a, b \in C(\mathscr{L})$, then $(a b)^{2}=(a b)(a b)=a b$ and therefore $a b \in C(\mathfrak{L})$. This proves the following theorem.

1.4. Theorem. For $a, b \in C(\&), a b=b a$ if and only if $a b \in C(\&)$.

2. Insets. A subset $\mathfrak{A}$ of the complete lattice $\mathscr{L}$ that contains $I$ and is closed under the intersection operation is called an inset (intersection-set) of $\mathfrak{L}$. The set of all insets of $\mathscr{L}$ will be designated by $I(\mathscr{L})$.

If $\left\{\mathfrak{A}_{i}\right\} \subset I(\mathscr{L})$, then the set-theoretic intersection $\bigcap_{i} \mathfrak{A}_{i}$ of the insets $\mathfrak{A}_{i}$ also is an inset. The union of the insets of this set is defined by

$$
\bigcup_{i} \mathfrak{A}_{i}=\left\{\bigcap_{i} A_{i} ; A_{i} \in \mathfrak{A}_{i}\right\} \text {. }
$$

Clearly $\bigcup_{i} \mathfrak{A}_{i}$ again is an inset of $\mathscr{L}$, and since $I \in \mathfrak{A}_{i}$ for each $i$, every $\mathfrak{A}_{i} \subset \bigcup_{i} \mathfrak{A}_{i}$. Actually, $U_{i} \mathfrak{A}_{i}$ is just the least inset of $\mathscr{L}$ containing every $\mathfrak{A}_{i}$. Thus $\{I(\mathscr{L})$; $\cup, \cap, \subset\}$ is a complete lattice with identity elements $\{I\}$ and $\&$ relative to $U$ and $\cap$ respectively.

For each $a \in Q(\mathcal{L})$, let $\mathscr{L}^{a}=\left\{A ; A \in \mathscr{L}, A=A^{a}\right\}$. If $\left\{A_{i}\right\} \subset \mathscr{L}^{a}$, then 
$\left(\bigcap_{i} A_{i}\right)^{a} \subset A_{i}^{a}=A_{i}$ and consequently $\left(\bigcap_{i} A_{i}\right)^{a} \subset \bigcap_{i} A_{i}$. Thus $\left(\bigcap_{i} A_{i}\right)^{a}=\bigcap_{i} A_{i}$ and we conclude that $\mathscr{L}^{a}$ is an inset of $\mathscr{L}$. Evidently $\mathscr{L}^{a}=\left\{A^{a} ; A \in \mathscr{L}\right\}$ if and only if $a \in C(\mathscr{L})$.

If $\mathfrak{A}$ is an inset of $\mathscr{L}$, then associated with each $A \in \mathfrak{L}$ is the least element $A^{\prime}$ of $\mathfrak{A}$ containing $A$. The mapping ': $A \rightarrow A^{\prime}, A \in \mathfrak{L}$, of $\mathscr{L}$ onto $\mathfrak{A}$ is a closure operation on $\mathscr{L}$. We shall designate this closure operation on $\mathscr{L}$ relative to $\mathfrak{A}$ by $\mathscr{L} / \mathfrak{A}$.

\subsection{TheOREM. For every $a \in Q(\mathcal{L}), \mathfrak{L} / \mathfrak{L}^{a}=a^{*}$.}

Proof. Let $b=\mathfrak{L} / \mathscr{L}^{a}$. Since $A^{b a}=A^{b}$ for each $A \in \mathfrak{L}, b a=b$ and $a \leqq b$ by 1.1. Clearly, then, $a^{*} \leqq b$. On the other hand, $o \leqq a \leqq a^{*}, a^{*} \leqq a^{*} a \leqq a^{*}$, and therefore $a^{*}=a^{*} a$. Hence $A^{a^{*}} \in \mathcal{L}^{a}$ for every $A \in \mathscr{L}$ and necessarily $A^{b} \subset A^{a^{*}}$ since $A^{b}$ is the least element of $\mathscr{L}^{a}$ containing $A$. Thus $b \leqq a^{*}$, and the theorem follows.

It is evident from this theorem that $\mathscr{L} / \mathscr{L}^{a}=a$ if and only if $a \in C(\mathscr{L})$. Also, for each $a \in Q(\mathscr{L})$ and $A \in \mathscr{L}, A^{a^{*}}$ is just the least element $B \supset A$ of $\mathscr{L}$ such that $B^{a}=B$.

2.2. Theorem. The mapping of the lattice $C(\mathscr{L})$ onto the lattice $I(\mathfrak{L})$ defined by the correspondence $a \rightarrow \mathscr{L}^{a}, a \in C(\mathscr{L})$, is a dual isomorphism.

Proof. We have $a \geqq b$ if and only if $a b=a$, and hence if and only if $\mathscr{L}^{a} \subset \mathscr{L}^{b}$.

As a consequence of this theorem, for every set $\left\{a_{i}\right\} \subset C(\mathscr{L})$, if $b=\cup_{i}^{*} a_{i}$, $\mathscr{L}^{b}=\bigcap_{i} \mathscr{L}^{a_{i}} ;$ if $c=\bigcap_{i} a_{i}, \mathscr{L}^{c}=\bigcup_{i} \mathscr{L}^{a_{i}}$.

For every $A, B \in \mathcal{L}$, let us define

$$
(\mathfrak{L} ; A, B)=\{C ; C \in \mathfrak{L}, C \cap A \subset B\} \text {. }
$$

Since $0 \in(\mathcal{L} ; A, B)$, each set $(\mathcal{L} ; A, B)$ is nonvoid. In view of the chain condition (C), Zorn's lemma may be applied to show that each set $(\mathscr{L} ; A, B)$ contains maximal elements. In fact, for every $C \in(\mathscr{L} ; A, B)$, there exists a maximal element of $(\mathscr{L} ; A, B)$ containing $C$.

A closure operation on $\mathscr{L}$ that is also an $\cap$-endomorphism of $\mathscr{L}$ will be called an $m$-closure operation on $\mathcal{L}$, and we shall designate the set of all $\mathrm{m}$ closure operations on $\mathscr{L}$ by $C_{m}(\mathfrak{L})$. Trivially $o$ and $i$ are in $C_{m}(\mathfrak{L})$.

2.3. ThEOREM. If $a \in C(\mathscr{L})$, then $a$ is an m-closure operation on $\mathfrak{L}$ if and only if, for each $A \in \mathfrak{L}$ and $B \in \mathcal{L}^{a}$, every maximal element of $(\mathfrak{L} ; A, B)$ is in $\mathfrak{L}^{a}$.

Proof. If $a \in C_{m}(\mathscr{L})$ and if $C$ is a maximal element of ( $\left.\mathscr{L} ; A, B\right), B \in \mathscr{L}^{a}$, then

$$
C^{a} \cap A \subset C^{a} \cap A^{a}=(C \cap A)^{a} \subset B^{a}=B .
$$

Hence, by the maximality of $C$ in $(\mathscr{L} ; A, B), C^{a}=C$ and $C \in \mathscr{L}^{a}$.

Conversely, let us assume that $a \in C(\mathscr{L})$ has the property that for each $A \in \mathfrak{L}$ and $B \in \mathscr{L}^{a}$ every maximal element of $(\mathfrak{L} ; A, B)$ is in $\mathfrak{L}^{a}$. Clearly 


$$
A \cap B \subset(A \cap B)^{a} \subset A^{a} \cap B^{a}
$$

for every $A, B \in \mathscr{L}$. If $C$ is a maximal element of $\left(\mathcal{L} ; B,(A \cap B)^{a}\right)$ that contains $A$, then $C \in \mathcal{L}^{a}$ by assumption. Hence $A^{a} \subset C$ and $A^{a} \cap B \subset(A \cap B)^{a}$. Thus $A^{a} \cap B^{a} \subset\left(A \cap B^{a}\right)^{a} \subset(A \cap B)^{a a}=(A \cap B)^{a}$, and therefore $A^{a} \cap B^{a}$ $=(A \cap B)^{a}$. Hence $a$ is an $m$-closure operation on $\mathscr{L}$.

For convenience, we shall call $\mathscr{L}^{a}$ an $m$-inset in case $a$ is an $m$-closure operation on $\mathcal{L}$. We shall designate by $I_{m}(\mathcal{L})$ the set of all $m$-insets of $\mathscr{L}$.

2.4. THEOREM. The set $I_{m}(\mathfrak{L})\left(C_{m}(\mathfrak{L})\right)$ is a complete sublattice of $I(\mathfrak{L})$ $(C(\mathscr{L}))$.

Proof. If $\left\{\mathfrak{H}_{i}\right\} \subset I_{m}(\mathcal{L})$ and if $C$ is a maximal element of $(\mathscr{L} ; A, B)$, where $B \in \bigcap_{i} \mathfrak{A}_{i}$, then $C \in \mathfrak{A}_{i}$ for each $i$ since $B \in \mathfrak{R}_{i}$ for each $i$. Hence $C \in \bigcap_{i} \mathfrak{A}_{i}$ and $\bigcap_{i} \mathfrak{A}_{i} \in I_{m}(\mathcal{L})$ by 2.3. If $a_{i}=\mathfrak{L} / \mathfrak{A}_{i}$, then $\bigcap_{i} a_{i}=\mathfrak{L} / \bigcup_{i} \mathfrak{P}_{i}$ by 2.2. Clearly $\bigcap_{i} a_{i} \in C_{m}(\mathfrak{L})$ and hence $\bigcup_{i} \mathfrak{A}_{i} \in I_{m}(L)$. This proves 2.4 .

Those insets of $\mathscr{L}$ that contain 0 are of special importance in our work. IVe shall designate by $I^{0}(\mathscr{L})$ the set of all such insets. Analogously, $C^{0}(\mathfrak{L})$ will designate the set of all closure operations $a$ on $\mathscr{L}$ such that $0^{a}=0$. For $\left\{\mathfrak{A}_{i}\right\} \subset I^{0}(\mathscr{L})$, clearly $\bigcup_{i} \mathfrak{A}_{i}$ and $\bigcap_{i} \mathfrak{A}_{i}$ are also in $I^{0}(\mathscr{L})$. Thus $I^{0}(\mathscr{L})\left(C^{0}(\mathscr{L})\right)$ is a complete sublattice of $I(\mathscr{L})(C(\mathscr{L}))$. A corollary of 2.4 is that $I_{m}^{0}(\mathscr{L})$ $\left(C_{m}^{0}(\mathscr{L})\right)$ is a complete sublattice of $I^{0}(\mathscr{L})\left(C^{0}(\mathscr{L})\right)$.

The $\cap$-identity element $d$ of $C^{0}(\mathfrak{L})$ clearly is defined by

$$
d: A^{d}=I, \quad A \neq 0 ; \quad 0^{d}=0 .
$$

In other words, $\{I, 0\}$ is the $\cup$-identity element of $I^{0}(\mathscr{L})$. It is not evident on the other hand what the $\cap$-identity element of $C_{m}^{o}(\mathscr{L})$ is in general. Evidently $o \in C_{m}^{0}(\mathscr{L})$, and, in some easily constructed examples, this is the only element of $C_{m}^{0}(\mathfrak{L})$. For a general ring, however, there are nontrivial examples of $m$-closure operations on the lattice of ideals of the ring, as we shall now show.

Let $R$ be a ring and $\mathcal{L}$ be the lattice of all ideals of $R$. An ideal $P$ of $R$ is prime [13] if $A B \subset P, A, B \in \mathcal{L}$, implies $A \subset P$ or $B \subset P$. If $\left\{P_{i}\right\}$ is a set of prime ideals of $R$ and $C=\bigcap_{i} P_{i}$, then $C$ is called a semi-prime ideal of $R$ [14]. In view of recent results of Levitzki $[11$, p. 29] and Nagata $[14$, Proposition 8], an ideal $C$ of $R$ is semi-prime if and only if $A^{2} \subset C, A \in \mathcal{L}$, implies $A \subset C$. We shall designate by $\mathscr{L}^{\pi}$ the set of all semi-prime ideals of $R$. Clearly $\mathfrak{L}^{\pi} \in I(\mathscr{L})$ and therefore $\pi \in C(\mathscr{L})$. The ring $R$ itself is called semi-prime if the zero ideal is semi-prime, that is, if $\pi \in C^{0}(\mathscr{L})$.

2.5. THEOREM. If $\mathscr{L}$ and $\mathfrak{L}^{x}$ are respectively the set of all ideals and the set of all semi-prime ideals of a ring $R$, then $\pi \in C_{m}(\mathfrak{L})$. Also, $\pi \in C_{m}^{0}(\mathfrak{L})$ if and only if $R$ is a semi-prime ring.

Proof. Let $A \in \mathfrak{L}, B \in \mathfrak{L}^{\pi}$, and $C$ be a maximal element of $(\mathscr{L} ; A, B)$. Then 
for every $D \in \mathscr{L}$ such that $D^{2} C C$, we have

$$
((D+C) \cap A)^{2} \subset A \cap C \subset B
$$

so that $(D+C) \cap A \subset B$. The maximality of $C$ implies that $D \subset C$. Thus $C \in \mathscr{L}^{\pi}$ and $\pi \in C_{m}(\mathcal{L})$ by 2.3 .

If $R$ is a semi-prime ring, $c$ defined by $A^{c}=A^{r l}, A \in \mathfrak{L}$, where $A^{r}\left(A^{l}\right)$ is the right (left) annihilator of $A$ in $R$, is a closure operation on $\mathscr{L}[10, \mathrm{p}$. 376]. That $c \in C_{m}^{0}(\mathscr{L})$ is a consequence of $[10,1.4]$. If $a \in C_{m}^{0}(\mathscr{L})$ and $A \in \mathscr{L}$, then $A \cap A^{r}=0\left[10\right.$, p. 376] and $A^{a} \cap A^{r}=0$. Thus $A^{a} \subset A^{r l}$ and $a \leqq c$. We conclude that $c$ is the unique maximal element (that is, the $\cap$-identity element) of $C_{m}^{0}(\mathcal{L})$.

3. Atomic insets. Any minimal nonzero element of a subset $\mathfrak{A}$ of the lattice $\mathfrak{L}$ is called an atom of $\mathfrak{A}$. If $\mathfrak{A}$ has an atom, $\mathfrak{A}$ is called an atomic subset of $\mathscr{L}$. Every inset $\mathfrak{L}^{a}$ of $\mathfrak{L}$ has a minimal element, namely $0^{a}$. If $a \notin C^{0}(\mathfrak{L})$, then trivially $\mathscr{L}^{a}$ is atomic with unique atom $0^{a}$. Otherwise, if $a \in C^{0}(\mathscr{L})$, the atoms of $\mathcal{L}^{a}$ are those elements of $\mathscr{L}^{a}$ which cover $0[2$, p. 5$]$, if such exist. We shall designate by $I_{a}(\mathscr{L})$ the set of all atomic insets of $\mathscr{L}$. If $L^{a} \in I_{a}(\mathscr{L})$, then we shall call $a$ an atomic closure operation on $\mathcal{L}$. We shall designate the set of all atomic closure operations on $\mathscr{L}$ by $C_{a}(\mathscr{L})$.

An atomic inset $\mathfrak{A}$ of $\mathfrak{L}$ is called homogeneous if for every nonzero $B \in \mathfrak{A}$ there exists an atom $A \in \mathfrak{A}$ such that $A \subset B$. If $\mathscr{L}^{a}$ is a homogeneous inset of $\mathfrak{L}, a$ is called a homogeneous closure operation on $\mathscr{L}$. The subsets of $I_{a}(\mathscr{L})$ and $C_{a}(\mathscr{L})$ composed of all the homogeneous elements will be designated by $I_{h}(\mathscr{L})$ and $C_{h}(\mathcal{L})$ respectively.

3.1. Lemma. If $a, b \in C_{m}(\mathscr{L})$ and $a$ is atomic (homogeneous), then $a \cup^{*} b$ is atomic (homogeneous).

Proof. Let $a \cup^{*} b=c$. The results are obvious if $0^{c} \neq 0$, so let us assume that $c \in C_{m}^{0}(\mathscr{L})$. For every atom $A \in \mathscr{L}^{a}$ we shall prove that $A^{c}$ is an atom of $\mathscr{L}^{c}$. Assume that $0 \neq B \subset A^{c}, B \in \mathscr{L}^{c}$. Since $(B \cap A)^{c}=B^{c} \cap A^{c}=B$, evidently $B \cap A$ $\neq 0$. However, $A$ is an atom of $\mathscr{L}^{a}$ while $B \in \mathscr{L}^{a}$, and therefore $B \cap A=A$. Hence $A \subset B$ and $A^{c} \subset B$, and we conclude that $A^{c}$ is an atom of $\mathscr{L}^{c}$.

If $a \in C_{h}(\mathscr{L})$ and $B \in \mathscr{L}^{c}, B \neq 0$, then there exists an atom $A \in \mathscr{L}^{a}$ such that $A \subset B$. Clearly $A^{c} \subset B$, and since $A^{c}$ is an atom of $\mathscr{L}^{c}, c \in C_{h}(\mathfrak{L})$.

An inspection of the proof of 3.1 shows us that no use is made of the assumption that $a$ and $b$ are $m$-closure operations other than that $a \cup^{*} b$ is an $m$-closure operation. Thus if $a \in C^{0}(\mathscr{L})$ and $b \in C_{m}^{0}(\mathscr{L})$, if $a$ is atomic, and if $a \leqq b$, then we can conclude from the above proof that $b$ also is atomic. However, if $a, b \in C^{0}(\mathscr{L})$, if $a$ is atomic, and if $a \leqq b$, then $b$ is not necessarily atomic. For example, a ring $R$ might have minimal right ideals so that its lattice $\mathfrak{L}$ of right ideals is atomic, and yet there might exist an infinite chain $R=A_{1} \supset A_{2} \supset \cdots$ of nonzero right ideals of $R$ having zero intersection. Evidently $\mathscr{L}^{b}=\left\{0, A_{1}, A_{2}, \cdots\right\}$ is a nonatomic inset such that $b \geqq o$, while 
$o$ is an atomic closure operation by assumption.

The situation relative to the intersection operation is more complex than that for the union operation given in 3.1. Thus it is not apparent for $a, b \in C_{m}(\mathscr{L}) \cap C_{h}(\mathscr{L})$ that $a \cap b$ is even atomic. For example, we might have $0^{a} \neq 0,0^{b} \neq 0$, and $0^{a} \cap 0^{b}=0$. In such a case, it is not clear that $\mathscr{L}^{a} \cup \mathscr{L}^{b}$ has atoms. However, a partial answer to this problem is given by the following lemma.

3.2. Lemma. If $a \in C_{m}^{0}(\mathscr{L}) \cap C_{h}(\mathscr{L})$ and $b \in C_{a}(\mathscr{L})$ then $a \cap b$ is atomic. If, furthermore, $b \in C_{m}^{0}(\mathscr{L}) \cap C_{h}(\mathscr{L})$ then $a \cap b$ is homogeneous.

Proof. Let $c=a \cap b$ so that $\mathscr{L}^{c}=\mathscr{L}^{a} \cup \mathscr{L}^{b}$. We shall prove that if $A \in \mathscr{L}^{a}$ and $B \in \mathcal{L}^{b}$ are atoms such that $A \cap B \neq 0$, then $A \cap B$ is an atom of $\mathscr{L}^{c}$. If, for $A^{\prime} \in \mathcal{L}^{a}$ and $B^{\prime} \in \mathscr{L}^{b}, 0 \neq A^{\prime} \cap B^{\prime} \subset A \cap B$ then clearly $A \cap A^{\prime} \neq 0$ and $B \cap B^{\prime} \neq 0$. Hence $A \subset A^{\prime}, B \subset B^{\prime}$, and $A \cap B \subset A^{\prime} \cap B^{\prime}$. Thus $A \cap B$ is an atom of $\mathscr{L}^{c}$.

In order to prove that $A \cap B \neq 0$ for some atoms $A \in \mathcal{L}^{a}$ and $B \in \mathcal{L}^{b}$, let $B$ be any atom of $\mathscr{L}^{b}$ and $A$ be an atom of $\mathscr{L}^{a}$ such that $A \subset B^{a}$. Since $(A \cap B)^{a}$ $=A \cap B^{a}=A \neq 0$ and $0^{a}=0$, evidently $A \cap B \neq 0$.

If, in addition, $b \in C_{m}^{0}(\mathcal{L}) \cap C_{h}(\mathcal{L})$, let $A^{\prime} \in \mathcal{L}^{a}$ and $B^{\prime} \in \mathcal{L}^{b}$ with $A^{\prime} \cap B^{\prime} \neq 0$. Select an atom $A \in \mathcal{L}^{a}$ such that $A \subset\left(A^{\prime} \cap B^{\prime}\right)^{a}=A^{\prime} \cap B^{\prime a}$. Since $\left(A \cap B^{\prime}\right)^{a}$ $=A \cap B^{\prime a}=A \neq 0, A \cap B^{\prime} \neq 0$. Next, choose an atom $B \in \mathscr{L}^{b}$ such that $B \subset\left(A \cap B^{\prime}\right)^{b}$. Since $B \subset A^{b}$ and $(A \cap B)^{b}=B \neq 0$, evidently $A \cap B \neq 0$. Clearly $A \cap B \subset A^{\prime} \cap B^{\prime}$, and we conclude that $a \cap b$ is homogeneous.

The following theorem is an immediate consequence of the preceding two lemmas.

3.3. Theorem. $C_{m}^{0}(\mathfrak{L}) \cap C_{h}(\mathscr{L})$ is a dual ideal of $C_{m}^{0}(\mathfrak{L})$.

4. Product of lattices. Associated with the lattices $\mathscr{L}$ and $\mathscr{T}$ is their direct product $\mathscr{L} \times \mathfrak{T}$. If $\mathscr{L}$ and $\mathfrak{T}$ are complete lattices satisfying $(C)$, then so is $\mathfrak{X} \times \mathfrak{T}$. For $(a, b) \in Q(\mathfrak{L}) \times Q(\mathfrak{T})$, let us define the mapping

$$
(a, b):(A, M)^{(a, b)}=\left(A^{a}, M^{b}\right), \quad A \in \mathcal{L}, M \in \mathfrak{T} .
$$

With this definition, $Q(\mathscr{L}) \times Q(\mathfrak{T}) \subset Q(\mathscr{L} \times \mathfrak{T})$, and, similarly, $C(\mathscr{L}) \times C(\mathfrak{T})$ $\subset C(\mathscr{L} \times \mathfrak{T})$ and $C_{m}(\mathscr{L}) \times C_{m}(\mathfrak{T}) \subset C_{m}(\mathscr{L} \times \mathfrak{T})$.

The projection mappings $\pi_{1}$ and $\pi_{2}$ of $\mathscr{L} \times \mathfrak{T}$ into $\mathscr{L}$ and $\mathfrak{T}$ respectively are defined as usual:

$$
\pi_{1}: \pi_{1}(A, M)=A ; \quad \pi_{2}: \pi_{2}(A, M)=M ; \quad(A, M) \in \mathcal{L} \times \mathfrak{T} .
$$

These mappings are lattice homomorphisms of $\mathscr{L} \times \mathfrak{T}$ onto $\mathscr{L}$ and $\mathfrak{T}$ respectively.

4.1. Lemma. $C_{m}(\mathfrak{L}) \times C_{m}(\mathfrak{T})=C_{m}(\mathfrak{L} \times \mathfrak{T})$.

Proof. For $c \in C_{m}(\mathscr{L} \times \mathfrak{T})$, we define the $m$-closure operations $a$ and $b$ on $\mathscr{L}$ and $\mathfrak{M}$ respectively by: 


$$
a: 1^{a}=\pi_{1}(A, I)^{c}, \quad A \in \mathcal{L} ; \quad b: M^{b}=\pi_{2}(I, M)^{c}, \quad M \in \mathfrak{T} .
$$

Since

$$
\begin{aligned}
(A, M)^{(a, b)} & =\left(A^{a}, M^{b}\right)=\left(A^{a}, I\right) \cap\left(I, M^{b}\right)=(A, I)^{c} \cap(I, M)^{c} \\
& =((A, I) \cap(I, M))^{c}=(A, M)^{c},
\end{aligned}
$$

clearly $c=(a, b)$. Thus $C_{m}(\mathscr{L} \times \mathfrak{T}) \subset C_{m}(\mathfrak{L}) \times C_{m}(\mathfrak{T})$ and 4.1 follows.

The inset of $\mathscr{L} \times \mathfrak{T}$ corresponding to $(a, b) \in C(\mathscr{L}) \times C(\mathfrak{T})$ evidently is $\mathscr{L}^{a} \times \mathfrak{M}^{b}$. This inset is atomic if and only if either $\mathcal{L}^{a}$ or $\mathfrak{T}^{b}$ is atomic; and it is homogeneous if and only if both $\mathfrak{L}^{a}$ and $\mathfrak{N}^{b}$ are homogeneous.

A mapping

$$
x: A \rightarrow x A, \quad A \in \mathfrak{L}, x A \in \mathfrak{M},
$$

of lattice $\mathcal{L}$ into lattice $\mathfrak{T}$ is called a $\cup$-map if and only if

1. $x 0=0$, and 2. $x\left(\cup_{i} A_{i}\right)=\bigcup_{i} x A_{i}$ for every set $\left\{A_{i}\right\} \subset \mathcal{L}$.

If

$1^{\prime} . x I=I$, and $2^{\prime} . x\left(\bigcap_{i} A_{i}\right)=\bigcap_{i} x A_{i}$ for every set $\left\{A_{i}\right\} \subset \mathcal{L}$, then $x$ is called a $\cap$-map of $\mathscr{L}$ into $\mathfrak{T}$. Either a $\cup$-map or a $\cap$-map of $\mathscr{L}$ into $\mathfrak{T}$ is order-preserving. We shall designate by $J(\mathscr{L} ; \mathfrak{T})$ and $K(\mathscr{L} ; \mathfrak{T})$ respectively the set of all $\cup$-maps and the set of all $\cap$-maps of $\mathcal{L}$ into $\mathfrak{T}$.

The sets $J(\mathscr{L} ; \mathfrak{T})$ and $K(\mathscr{L} ; \mathfrak{T})$ are partially-ordered in the usual way: $x \leqq y$ if and only if $x A \subset y A$ for every $A \in \mathscr{L}$. The set $J(\mathscr{L} ; \mathfrak{T})$ is closed under the union operation defined by:

$$
\bigcup_{i} x_{i}:\left(\bigcup_{i} x_{i}\right) A=\bigcup_{i}\left(x_{i} A\right), \quad A \in \mathcal{L},\left\{x_{i}\right\} \subset J(\mathcal{L} ; \mathfrak{T}) .
$$

Similarly, $K(\mathfrak{L} ; \mathfrak{T})$ is closed under the intersection operation defined by:

$$
\bigcap_{i} x_{i}:\left(\bigcap_{i} x_{i}\right) A=\bigcap_{i}\left(x_{i} A\right), \quad A \in \mathscr{L},\left\{x_{i}\right\} \subset K(\mathfrak{L} ; \mathfrak{T}) .
$$

For $x \in J(\mathscr{L} ; \mathfrak{T})$ and $M \in \mathscr{N}$, let $\left\{A_{i}\right\}$ be the set of all $A_{i} \in \mathscr{L}$ such that $x A_{i} \subset M$. Since $x\left(\bigcup_{i} A_{i}\right) \subset M$ also, there is a unique maximal element $A \in \mathscr{L}$ such that $x A \subset M$. We designate this element by $x^{-1} M$. Clearly $x^{-1}$ may be thought of as a mapping of $\mathfrak{M}$ into $\mathcal{L}$ :

$$
x^{-1}: x^{-1} M=\max A \text { such that } x A \subseteq M, M \in \Re \text {. }
$$

It is evident that for every $M \in \mathscr{N}$ and $A \in \mathscr{L}$,

$$
\begin{aligned}
& x\left(x^{-1} M\right) \subset M ; \quad x^{-1}(x A) \subset A ; \\
& x A \subset M \text { if and only if } A \subset x^{-1} M .
\end{aligned}
$$

Dually, for $x \in K(\mathscr{L} ; \mathfrak{T})$ and $M \in \mathscr{T}$, there exists a unique minimal ele- 
ment $A \in \mathfrak{L}$ such that $x A \supset M$. We designate this element also by $x^{-1} M$. Thus

$$
x^{-1}: x^{-1} M=\min A \text { such that } x A \supset M, \quad M \in \mathscr{T},
$$

is a mapping of $\mathscr{T}$ into $\mathscr{L}$. In this case, for every $M \in \mathfrak{T}$ and $A \in \mathfrak{L}$,

$$
\begin{aligned}
& x\left(x^{-1} M\right) \supset M ; \quad x^{-1}(x A) \subset A ; \\
& x A \supset M \text { if and only if } A \supset x^{-1} M .
\end{aligned}
$$

4.2. Lemma. If $x$ is a $\cup$-map $(\cap-m a p)$ of $\mathcal{L}$ into $\mathfrak{T}$ then $x^{-1}$ is a $\cap$-map $(\cup-m a p)$ of $\mathfrak{M}$ into $\mathscr{L}$.

Proof. If $\left\{M_{i}\right\} \subset \Re, A=x^{-1}\left(\bigcap_{i} M_{i}\right)$, and $B=\bigcap_{i} x^{-1} M_{i}$, then $x A \subset \bigcap_{\imath} M_{v}$, $x A \subset M_{i}$ for each $i, A \subset x^{-1} M_{i}$ for each $i$, and therefore $A \subset B$. On the other hand, $B \subset x^{-1} M_{i}$ and $x B \subset M_{i}$ for each $i$ so that $x B \subset \cap_{i} M_{i}$ and $B \subset A$. Hence $A=B$. Clearly $x^{-1} I=I$, and $x^{-1} \in K(\mathfrak{T} ; \mathcal{L})$. The dual is proved similarly.

4.3. Lemma. If $x$ is a $\cup_{-m a p}(\cap-m a p)$ of $\mathcal{L}$ into $\mathfrak{T l}$, then $x=\left(x^{-1}\right)^{-1}$.

Proof. By definition, $\left(x^{-1}\right)^{-1} A=\cap M, x^{-1} M \supset A$. Hence $\left(x^{-1}\right)^{-1} A=\cap M$, $x A \subset M$, and $\left(x^{-1}\right)^{-1} A=x A$. The proof of the dual is similar.

4.4. THEOREM. The mapping ${ }^{-1}: x \rightarrow x^{-1}$ is an isomorphism between the algebraic systems $\{J(\mathfrak{L} ; \mathfrak{T}) ; \leqq, \cup\}$ and $\{K(\mathfrak{T T} ; \mathfrak{L}) ; \geqq, \cap\}$.

Proof. If $x, y \in J(\mathfrak{L} ; \mathfrak{T})$ with $x \leqq y$, then $x\left(y^{-1} M\right) \subset y\left(y^{-1} M\right) \subset M$ and $y^{-1} M \subset x^{-1} M$ for every $M \in \Re$. Thus $x^{-1} \geqq y^{-1}$. If $x^{-1}=y^{-1}$, then $\left(x^{-1}\right)^{-1}$ $=\left(y^{-1}\right)^{-1}$ and $x=y$ by 4.3 . Clearly, then, the mapping ${ }^{-1}: x \rightarrow x^{-1}$ is a $1-1$ order inverting mapping between $J(\mathscr{L} ; \mathfrak{T})$ and $K(\mathfrak{T} ; \mathfrak{L})$. Such a mapping carries least upper bounds into greatest lower bounds and vice versa.

A $\cap$-map $x$ of $\mathscr{L}$ into $\mathfrak{T}$ is called a $\cap^{\prime}-m a p$ if and only if

$$
x\left(\bigcup_{i} A_{i}\right)=\bigcup_{i}\left(x A_{i}\right) \text { for every chain }\left\{A_{i}\right\} \subset \mathcal{L} .
$$

We shall designate by $K^{\prime}(\mathscr{L} ; \mathfrak{T})$ the set of all $\cap^{\prime}$-maps of $\mathscr{L}$ into $\mathfrak{T}$.

4.5. Theorem. If $x, y \in K^{\prime}(\mathscr{L} ; \mathfrak{T})$, then also $x \cap y \in K^{\prime}(\mathscr{L} ; \mathfrak{M})$.

Proof. Let $\left\{A_{i}\right\}$ be a chain in $\mathscr{L}$. Then $(x \cap y)\left(\cup_{i} A_{i}\right)=x\left(\bigcup_{i} A_{i}\right) \cap y\left(\cup_{i} A_{i}\right)$ $=\left(\cup_{i} x A_{i}\right) \cap\left(\cup_{i} y A_{i}\right)$. Since $\left\{x A_{i}\right\}$ and $\left\{y A_{i}\right\}$ are chains in $\mathscr{T}$, the chain condition (C) implies that $\left(\mathrm{U}_{i} x A_{i}\right) \cap\left(\mathrm{U}_{i} y A_{i}\right)=\mathrm{U}_{i}\left(\mathrm{U}_{j}\left(x A_{i} \cap y A_{j}\right)\right)$. If, for indices $i$ and $j, A_{i} \subset A_{j}$, then $x A_{\mathfrak{r}} \cap y A_{j} \subset x A_{j} \cap y A_{j}$; therefore

$$
\bigcup_{i}\left(\bigcup_{j}\left(x A_{i} \cap y A_{j}\right)\right) \subset \bigcup_{i}\left(x A_{i} \cap y A_{i}\right) .
$$

Since the reverse inequality trivially is true, evidently $(x \cap y)\left(\cup_{i} A_{i}\right)=U_{i}$ $\left(x A_{i} \cap y A_{i}\right)=U_{i}(x \cap y) A_{i}$, and 4.5 follows.

For every $x \in J(\mathscr{L} ; \mathfrak{T})$, the mapping $x^{-1} x$ is easily verified to be a closure operation on $\mathscr{L}$. Actually, the following more general result holds. 
4.6. Theorem. If $x \in J(\& ; \mathfrak{T})$ and $c \in C(\mathfrak{T})$, then $x^{-1} c x \in C(\mathscr{L})$.

Proof. Since $\left(x^{-1} c x\right) A=x^{-1}(x A)^{c} \supset x^{-1}(x A) \supset A$ for every $A \in \mathcal{L}$, clearly $x^{-1} c x \in Q(\mathfrak{L})$. If $\left(x^{-1} c x\right) A=B$, then $x B \subset(x A)^{c},(x B)^{c} \subset(x A)^{c}$, and $\left(x^{-1} c x\right) B$ $\subset\left(x^{-1} c x\right) A=B$. Hence $x^{-1} c x \in C(\mathscr{L})$.

4.7. Theorem. If $x \in J(\mathfrak{L} ; \mathfrak{M}), a \in C(\mathfrak{L})$, and $b \in C(\mathfrak{T})$, then $a \leqq x^{-1} b x$ if and only if $x^{-1} \mathfrak{M}^{b} \subset \mathfrak{L}^{a}$.

Proof. The condition $x^{-1} \mathfrak{N}^{b} \subset \mathcal{L}^{a}$ is equivalent to $a x^{-1} b=x^{-1} b$. If $a x^{-1} b$ $=x^{-1} b$, then $a x^{-1} b x=x^{-1} b x$ and $a \leqq x^{-1} b x$ by 1.1. Conversely, if $a \leqq x^{-1} b x$, then $x^{-1} b \leqq a x^{-1} b \leqq x^{-1} b x x^{-1} b \leqq x^{-1} b$ and $x^{-1} b=a x^{-1} b$. This proves 4.7.

For the $\cap^{\prime}$-map $x$ of $\mathscr{L}$ into $\mathfrak{M}$, let us define

$$
(\mathscr{L} ; x, M)=\{A ; A \in \mathscr{L}, x \mathcal{A} \subset M\}, \quad M \in \mathfrak{N} .
$$

Obviously $(\mathscr{L} ; x, M)$ is nonvoid if and only if $x 0 \subset M$. If $x 0 \subset M$, then $(\mathscr{L} ; x, M)$ has maximal elements by Zorn's lemma. Actually, for every $A \in(\mathfrak{L} ; x, M)$, there exists at least one maximal element of $(\mathscr{L} ; x, M)$ containing $A$.

4.8. Theorem. If $x \in K^{\prime}(\mathfrak{T} ; \mathfrak{L}), a \in C(\mathfrak{L})$, and $b \in C(\mathfrak{T})$, then $a x=x b$ if and only if (1) $a \leqq x b x^{-1}$, and (2) for every $A \in \mathscr{L}^{a}$ such that $A \supset x 0$, the maximal elements of $(\mathfrak{T} ; x, A)$ are contained in $\mathfrak{T}^{b}$.

Proof. If $a x=x b$, then $a x b x^{-1}=x b x^{-1}$ and $a \leqq x b x^{-1}$. For $A \in \mathscr{L}^{a}$ such that $A \supset x 0$, let $M$ be a maximal element of $(\mathscr{T} ; x, A)$. Since $x M \subset A, x M^{b}=\left(x M^{a}\right)$ $\subset A^{a}=A$ and $M^{b}=M$ due to the maximality of $M$. Hence $M \in \mathbb{N}^{b}$.

Conversely, if (1) and (2) are satisfied, $x M \subset a x M$ and therefore $x b M$ Cax $M$ since the maximal elements of $(\mathfrak{T} ; x, a x M)$ containing $M$ are in $\mathfrak{N}^{b}$. However, $a x M \subset\left(x b x^{-1}\right) x M \subset x b M$; hence $a x M=x b M$ for every $M \in \mathscr{T}$ and $a x=x b$.

4.9. Theorem. If $x \in K^{\prime}(\mathfrak{T} ; \mathfrak{L})$, the set $\mathfrak{C}$ of all $(a, b) \in C(\mathscr{L}) \times C(\mathfrak{T})$ such that $a x=x b$ is a complete sublattice of $\widehat{C}(\mathfrak{L}) \times C(\mathfrak{T})$.

Proof. Assume that $\left\{\left(a_{i}, b_{i}\right)\right\} \subset \mathcal{C}$. Then for every $M \in \mathfrak{M}$,

$$
\left(\bigcap_{i} a_{i}\right) x M=\bigcap_{i}\left(a_{i} x M\right)=\bigcap_{i}\left(x b_{i}\right) M=x\left(\bigcap_{i} b_{i}\right) M .
$$

Hence $\cap_{i}\left(a_{i}, b_{i}\right) \in \mathcal{C}$. If $a=\bigcup_{i}^{*} a_{i}$ and $x M=A$, then $A^{a}=B$ where $B$ is the minimum element of $\mathscr{L}$ such that $B \supset A$ and $\cup_{i} B^{a_{i}}=B$, that is, such that $B \supset A$ and $B^{a_{i}}=B$ for each $i$ (see 2.1). Similarly, if $b=\cup_{i}^{*} b_{i}, M^{b}=N$ where $N$ is the minimum element of $\mathscr{T}$ such that $N \supset M$ and $N^{b_{i}}=N$ for each $i$. Since $x N \supset A$ and $a_{i} x N=x b_{i} N=x N$ for each $i$, evidently $x N \supset B$. On the other hand, $x M \subset B$; and if $M_{1}$ is a maximum element of $(\mathfrak{T} ; x, B)$ containing $M$, then $M_{1}^{b_{i}}=M_{1}$ for each $i$ and $M_{1} \supset N$. Hence $x N \subset B$, which together with a previous inequality proves that $x N=B$. Therefore $x b M=x N=B=A^{a}$ $=a x M$ for every $M \in \mathscr{N}$ and $a x=x b$. Thus $\bigcup_{i}^{*}\left(a_{i}, b_{i}\right) \in \mathfrak{e}$, and 4.9 is proved. 
In many of the applications to ring theory, each mapping $x$ of $K^{\prime}(\mathfrak{T} ; \mathfrak{L})$ has the following property.

(P) For each $A \in \mathscr{L}$ and $M \in \mathscr{T}$, there exists $B \subset A$ such that

$$
x^{-1} A \cap M=x^{-1} B \text {. }
$$

4.10. Theorem. Let $x \in K^{\prime}(\mathfrak{T} ; \mathfrak{L})$ satisfy property $(\mathrm{P})$. If $a \in C_{a}(\mathfrak{L})$ and $b \in C_{m}^{0}(\mathfrak{T})$ such that $a \leqq x b x^{-1}$, and if there exists an atom $A \in \mathscr{L}^{a}$ such that $x^{-1} A \neq 0$, then $b$ is atomic and $\left(x^{-1} A\right)^{b}$ is an atom of $\mathfrak{M}^{b}$.

Proof. If $0 \neq M \subset\left(x^{-1} A\right)^{b}$ for $M \in \mathfrak{N}^{b}$, then $M \cap x^{-1} A \neq 0$ since $\left(M \cap x^{-1} A\right)^{b}$ $=M$. Hence, by $(\mathrm{P})$, there exists $B \subset A, B \neq 0$, such that $M \cap x^{-1} A=x^{-1} B$. Thus $B \subset x M \cap A$. By 4.7, $x M \in \mathscr{L}^{a}$ and therefore, since $A$ is an atom of $\mathscr{L}^{a}$, $A \subset x M$ and $x^{-1} A \subset M$. Hence $\left(x^{-1} A\right)^{b} \subset M$ and we conclude that $\left(x^{-1} A\right)^{b}$ is an atom of $\mathbb{T}^{b}$.

5. Ring-theoretic applications. We shall give some simple applications of our results to ring theory in this section, leaving the more complicated applications to the next paper.

Associated with the ring $R$ is its lattice $\mathscr{L}$ of all right ideals. For each subset $A$ of $R$, let $A^{l}\left(A^{r}\right)$ designate the left (right) annihilator of $A$ in $R$. Since $A^{l}=A^{l r l}$ for each $A \in \mathcal{L}$, it is evident that $l r$ is a closure operation on $\mathcal{L}$.

We shall assume that $R$ is a faithful ring in this section, that is, that $R$ is a ring such that $R^{l}=0$. It will be convenient to call an ideal $S$ of $R$ large if $S^{l}=0$. A right ideal $A$ of $R$ will be called prime $[7 ; 10]$ if and only if :

$$
r S \subset A, r \in R \text { and } S \text { a large ideal of } R \text {, implies } r \in A \text {. }
$$

Let us designate by $\mathscr{L}^{p}$ the set of all prime right ideals of $R$. It is evident that the set $\mathfrak{L}^{p}$ is closed under the intersection operation, and hence that $p \in C(\mathscr{L})$.

If $A \in \mathcal{L}$ and $r S \subset A^{\text {lr }}$ for some $r \in R$ and large ideal $S$ of $R$, then $A^{l^{r}} S=0$ and $A^{l_{r}=0}$. Thus $r \in A^{l r}$, and we conclude that $A^{l_{r}} \in \mathscr{L}^{p}$. Hence $p \leqq l r$.

\subsection{TheOREM. $p \in C_{m}^{0}(\mathscr{L})$.}

Proof. Let $A \in \mathfrak{L}, B \in \mathfrak{L}^{p}$, and $C$ be a maximal element of $(\mathfrak{L} ; A, B)$. Assume that $a S \subset C$ for some $a \in R$ and some large ideal $S$ of $R$. If $(a)_{r}$ designates the $r$-ideal of $R$ generated by $a,\left[\left((a)_{r}+C\right) \cap A\right] S \subset C \cap A \subset B$, and $\left((a)_{r}+C\right)$ $\cap A \subset B$ since $B$ is prime. Thus $(a)_{r} \subset C$ and $a \in C$ due to the maximality of $C$ in $(\mathscr{L} ; A, B)$. Hence $C$ is prime, and $p$ is an $m$-closure operation on $\&$ by 2.3 . Clearly $0 \in \mathcal{L}^{p}$ and 5.1 is proved.

This theorem was proved for semi-prime rings in $[10,2.8]$ by a different method.

A right $R$-module will be called prime if and only if

$$
x S=0, x \in M \text { and } S \text { a large ideal of } R \text {, implies } x=0 .
$$

For example, if $A$ is a prime right ideal of the faithful ring $R$, both $A$ and 
$R-A$ are prime as right $R$-modules. We shall assume henceforth in this section that $M$ is a prime right $R$-module. Let $\Re$ designate the lattice of all submodules of $M$. A submodule $N$ of $M$ will be called prime if and only if $x S \subset N, x \in M$ and $S$ a large ideal of $R$, implies $x \in N$.

If $\mathscr{T}^{p}$ designates the set of all prime submodules of $M$, then it may be shown by the same proof as in 5.1 that $p \in C_{m}^{0}(\mathfrak{T})$.

For every $x \in M$ and $A \in \mathcal{L}, x A$ is a submodule of $M$. Since $x\left(\bigcup_{i} A_{i}\right)$ $=U_{i} x A_{i}$ for every set $\left\{A_{i}\right\} \subset \mathscr{L}$, we may consider $x$ as a $\bigcup_{\text {-map of }} \mathscr{L}$ into $\mathfrak{T}$. The inverse $\cap$-map $x^{-1}$ of $\mathfrak{T}$ into $\mathscr{L}$ may be defined as follows:

$$
x^{-1}: x^{-1} N=\{a ; a \in R, x a \in N\},
$$

$N \in \Re$.

If $\left\{N_{i}\right\}$ is a chain in $\mathscr{M}$ and $N=\cup_{i} N_{i}$, and if $a \in x^{-1} N$, then $x a \in N$ and $x a \in N_{i}$ for some $i$. Clearly, then, $x^{-1}\left(\bigcup_{i} N_{i}\right)=\bigcup_{i} x^{-1} N_{i}$, and thus $x^{-1}$ is a $\cap^{\prime}$-map of $\mathfrak{T}$ into $\&$.

It is natural to ask what the least prime right ideal is that contains $x^{-1} N$ for $x \in M$ and $N \in \mathscr{T}$. For the special case in which $R$ is a semi-prime ring, the answer is known to be that $[10,2.13]\left(x^{-1} N\right)^{p}=x^{-1} N^{p}$. That this is the answer for every faithful ring $R$ and every prime right $R$-module $M$ is seen by the following theorem.

\subsection{Theorem. For every $x \in M, p x^{-1}=x^{-1} p$.}

Proof. If $N \in \mathscr{T N}^{p}$ and $a S \subset x^{-1} N, a \in R$ and $S$ a large ideal of $R$, then $x a S \subset N$ and $x a \in N$. Thus $a \in x^{-1} N$ and we conclude that $x^{-1} N \in \mathscr{L}^{p}$. Since $x^{-1} \mathfrak{M}^{p} \subset \mathcal{L}^{p}, p \leqq x^{-1} p x$ by 4.7. Next, let $A \in \mathscr{L}^{p}$ and $x \in M$ be such that $A \supset x^{-1} 0$, and let $N$ be a maximal element of $\left(\Re ; x^{-1}, A\right)$. If $y S \subset N$ for $y \in M$ and $S$ a large ideal of $R$, and if $N^{\prime}$ is the submodule of $M$ generated by $N$ and $y$, evidently $N^{\prime} S \subset N$. Now $x\left(x^{-1} N^{\prime}\right) \subset N^{\prime}, x\left(x^{-1} N^{\prime}\right) S \subset N^{\prime} S \subset N$, and $\left(x^{-1} N^{\prime}\right) S$ $\subset x^{-1} N \subset A$. Thus, since $A$ is prime, $x^{-1} N^{\prime} \subset A$. The maximality of $N$ implies that $N=N^{\prime}$, and therefore $y \in N$. Thus $N$ is prime, and we conclude that all the maximal elements of $\left(\mathfrak{T} ; x^{-1}, A\right), A \in \mathcal{L}^{p}$, are in $\mathfrak{T}^{p}$. The desired conclusion now follows from 4.8 .

6. Modular lattices. We now further restrict the lattices under consideration to be modular. Thus let $\&$ be a complete modular lattice satisfying the chain condition (C). All the ideal lattices associated with a ring are of this type.

For each $a \in C(\mathscr{L})$, the inset $\mathscr{L}^{a}$ can be made into a lattice by defining the union operation in the obvious way:

$$
\mathrm{V}: \bigvee_{i} A_{i}=\left(\bigcup_{i} A_{i}\right)^{a}, \quad\left\{A_{i}\right\} \subset \mathcal{L}^{a}
$$

Evidently 


$$
\left(\mathrm{U} A_{i}\right)^{a}=\mathrm{V}_{i} A_{i}^{a} \text { for every set }\left\{A_{i}^{a}\right\} \subset \mathcal{L} .
$$

Of course, the lattice $\left\{\mathscr{L}^{a} ; \cap, \vee, \subset\right\}$ is not in general a sublattice of $\mathscr{L}$.

6.1. TheOREM. If $a \in C_{m}(\mathscr{L}), \mathscr{L}^{a}$ is a complete modular lattice satisfying condition (C).

Proof. If $A, B, C \in \mathfrak{L}$ with $A \subset C$, then $(A \cup B)^{a} \cap C^{a}=(A \cup(B \cap C))^{a}$ and $\left(A^{a} \bigvee B^{a}\right) \cap C^{a}=A^{a} \bigvee\left(B^{a} \cap C^{a}\right)$. Hence $\mathcal{L}^{a}$ is modular. If $\left\{A_{i}\right\}$ is a chain in $\mathcal{L}^{a}$ and $A \in \mathcal{L}^{a}$, then $\left(\mathrm{V}_{i} A_{i}\right) \cap A=\left(\mathrm{U}_{i} A_{i}\right)^{a} \cap A=\left(\left(\mathrm{U}_{i} A_{i}\right) \cap A\right)^{a}=\left(\mathrm{U}_{i}\left(A_{i} \cap A\right)\right)^{a}$ $=\mathrm{V}_{\boldsymbol{i}}\left(A_{\boldsymbol{i}} \cap A\right)^{a}=\mathrm{V}_{\boldsymbol{i}}\left(A_{i} \cap A\right)$. Thus $\mathscr{L}^{a}$ satisfies $(\mathrm{C})$.

A complete modular lattice satisfying $(C)$ is complemented if and only if the following condition (D) is satisfied:

(D) For every $A \in \mathscr{L}, A \neq I$, there exists some $B \in \mathcal{L}, B \neq 0$, such that $A \cap B=0$.

To prove this, assume that (D) holds in $\mathscr{L}$. For $A \in \mathfrak{L}, A \neq I$, let $B$ be a maximal element of $(\mathfrak{L} ; A, 0)$. If $A \cup B \neq I$, there exists a nonzero $C \in \mathfrak{L}$ such that $(A \cup B) \cap C=0$. Then it is easily shown that $A \cap(B \cup C)=0$ contrary to the maximality of $B$. Hence $A \cup B=I$.

Let us call the closure operation $a \in C^{0}(\mathscr{L})$ reducible if for every $A \in \mathfrak{L}^{a}$, $A \neq I$, there exists some $B \in \mathcal{L}, B \neq 0$, such that $A \cap B=0$. The following theorem is a consequence of 6.1 and our remarks above.

6.2. TheOREM. If $a \in C_{m}^{0}(\mathfrak{L})$, then $a$ is reducible if and only if $\mathfrak{L}^{a}$ is a complemented modular lattice.

The center $\mathfrak{C}$ of a complemented modular lattice $\mathfrak{L}$ is the set of all elements of $\mathscr{L}$ having unique complements [2, p. 124]. It is well-known that $\mathfrak{C}$ is a sublattice of $\mathscr{L}$ that is a Boolean algebra. If the lattice $\mathscr{L}$ is complete, then so is $\mathbb{C}$.

An element $B \in \mathfrak{L}$ is said to be an essential extension of $A \in \mathfrak{L}$ if $A \subset B$, and for every $C \in \mathscr{L}$ such that $C \cap B \neq 0$ also $C \cap A \neq 0$. If $B$ is an essential extension of $A$, we shall write $A \subset^{\prime} B$. For each $A \in \mathscr{L}$, let $\mathcal{E}(A)=\{B ; B \in \mathcal{L}$, $\left.A C^{\prime} B\right\}$. If $\left\{B_{i}\right\}$ is a chain contained in $\mathcal{E}(A)$, then clearly $\mathrm{U}_{i} B_{i} \in \mathcal{E}(A)$. Thus $\mathcal{E}(A)$ has maximal elements by Zorn's lemma. If $B \in \mathcal{E}(A)$, then $\mathcal{E}(B)$ $C \mathcal{E}(A)$; and if $B$ is a maximal element of $\mathcal{E}(A), \mathcal{E}(B)=\{B\}$.

If $A, B \in \mathcal{L}$ and $A \cap B=0$, then $A^{\prime} \cap B^{\prime}=0$ for every $A^{\prime} \in \mathcal{E}(A)$ and $B^{\prime} \in \mathcal{E}(B)$. On the other hand, if $A \in \mathfrak{L}$ and $B$ is a maximal element of $(\mathfrak{L} ; A, 0)$, then for every $C$ such that $A \subset C$ and $C \cap B=0$ necessarily $A C^{\prime} C$. To prove this, let $D \in \mathcal{L}, 0 \neq D \subset C$. Then $A \cap(B \cup D) \neq 0$, and, since $C \cap(B \cup D)=D$, $A \cap D \subset A \cap(B \cup D) \subset A \cap D$. Thus $A \cap D \neq 0$, and $A C^{\prime} C$. If $B$ is a maximal element of $(\mathfrak{L} ; A, 0), I$ is the unique maximal element of $\mathcal{E}(A \cup B)$.

6.3. THEOREM. If the lattice $\mathfrak{L}$ has a reducible $m$-closure operation a, then a 
is unique and is the $\cap$-identity element of $C_{m}^{0}(\mathfrak{L})$, Also, for each $A \in \mathfrak{L}, A^{a}$ is the unique maximal essential extension of $A$ in $\mathfrak{L}$.

Proof. If $a$ is reducible and is in $C_{m}^{0}(\mathcal{L})$, and if $A \in \mathscr{L}$ is such that $A \cap B \neq 0$ for every nonzero $B \in \mathcal{L}$, then $A^{a}=I$. If $A \in \mathcal{L}$ and $A \cap B=0$ for some nonzero $B \in \mathcal{L}$, select $B$ as a maximal element of ( $\mathcal{L} ; A, 0)$. Clearly $B \in \mathcal{L}^{a}$. Since $A^{a} \cap B=0, A^{a} \in \mathcal{E}(A)$ by previous remarks. Also, $A^{a} \bigvee B=I$ by 6.2 . If $C$ is a maximal element of $\mathcal{E}(A)$, then $C \in \mathscr{L}^{a}$ and $C \supset A^{a}$. Thus $C \vee B=I$ and $C=C \cap I=C \cap\left(A^{a} \bigvee B\right)=A^{a} \bigvee(C \cap B)=A^{a}$. Consequently $A^{a}$ is the unique maximal element of $\mathcal{E}(A)$, and $a$ itself is unique. If $b \in C_{m}^{0}(\mathfrak{L})$ and $b \geqq a$, then $\mathscr{L}^{b} \subset \mathscr{L}^{a}$ and therefore $b$ is reducible. Hence $a$ is the maximal element of $C_{m}^{0}(\mathscr{L})$, namely the $\cap$-identity element.

An atomic lattice has been defined in [2, p. 130] as a complete modular lattice satisfying condition (C) and having atoms, with the union of the atoms of the lattice being $I$. Every atomic lattice is complemented, and each nonzero element of the lattice is a union of atoms.

6.4. TheOREM. Let a be a reducible $m$-closure operation on $\mathfrak{L}$, and let $\mathfrak{L}^{c}$ be the center of $\mathfrak{L}^{a}$. If $a$ is atomic then so is $c$, whereas if $a$ is homogeneous then both $\mathscr{L}^{a}$ and $\mathscr{L}^{c}$ are atomic lattices.

Proof. If $A$ is an atom of $\mathscr{L}^{a}$ and $0 \neq B \subset A^{c}, B \in \mathscr{L}^{c}$, then $A \cap B \neq 0$. For if $A \cap B=0$, then the complement $B^{\prime}$ of $B$ must contain $A$, and therefore $A \subset B^{\prime} \cap A^{c} \neq A^{c}$ contrary to the fact that $A^{c}$ is the least element of $\mathscr{L}^{c}$ containing $A$. Since $A \cap B \neq 0, A \subset B$ and $A^{c} \subset B$. Thus $A^{c}$ is an atom of $\mathscr{L}^{c}$.

If $a$ is homogeneous and $C$ is the union of the atoms of $\mathscr{L}^{a}$, then $\left(\mathscr{L}^{a} ; C, 0\right)$ $=\{0\}$ since otherwise it would contain an atom of $\mathscr{L}^{a}$. Thus $C=I$ and $\mathscr{L}^{a}$ is an atomic lattice by 6.1. The union of the atoms of $\mathscr{L}^{c}$ also is $I$, and $\mathscr{L}^{c}$ is an atomic lattice.

An atomic lattice $\mathscr{L}$ is said to be simple if $\{0, I\}$ is the center of $\mathscr{L}$. The atoms of a simple lattice $\mathscr{L}$ may be taken to be the points of a projective geometry [2, p. 116]. For this reason, a simple lattice is often referred to as a projective geometry.

If now $a$ is a homogeneous, reducible $m$-closure operaton on the lattice $\mathscr{L}$, so that $\mathscr{L}^{a}$ and $\mathscr{L}^{c}$ are atomic lattices, then for each atom $C$ of $\mathscr{L}^{c}$ let $\odot(C)$ $=\left\{A ; A \in \mathscr{L}^{a}, A \subset C\right\}$. Clearly $\odot(C)$ is a simple lattice. If $\left\{C_{i}\right\}$ is the set of all atoms of $\mathscr{L}^{c}$, then $\mathcal{L}^{a}=\sum_{i}^{*} \mathcal{P}\left(C_{i}\right)$, where $\sum^{*}$ designates the full direct union. Stated otherwise, this is the well-known result that an atomic lattice $\mathscr{L}^{a}$ is a full direct union of projective geometries.

\section{BIBLIOGRAPHY}

1. R. Baer, Abelian groups that are direct summands of every containing abelian group, Bull. Amer. Math. Soc. vol. 46 (1940) pp. 800-806.

2. G. Birkhoff, Lattice theory, Amer. Math. Soc. Colloquium Publications, rev. ed., vol. 25, New York, 1948. 
3. R. L. Blair, Ideal lattices and the structure of rings, Trans. Amer. Math. Soc. vol. 75 (1953) pp. 136-153.

4. B. Eckmann and A. Schopf, Über injektive Moduln, Archive der Mathematik vol. 4 (1953) pp. 75-78.

5. S. Hanai, On commutative T-closure operators, Kodai Math. Sem. Rep. (1953) pp. 17-19, Math. Rev. vol. 15 (1954) p. 193.

6. R. E. Johnson, The extended centralizer of a ring over a module, Proc. Amer. Math. Soc. vol. 2 (1951) pp. 891-895.

7. - Prime rings, Duke Math. J. vol. 18 (1951) pp. 799-809.

8. _ - Representations of prime rings, Trans. Amer. Math. Soc. vol. 74 (1953) pp. 351-357.

9. - The imbedding of a ring as an ideal in another ring, Duke Math. J. vol. 20 (1953) pp. 569-574.

10. - Semi-prime rings, Trans. Amer. Math. Soc. vol. 76 (1954) pp. 375-388.

11. J. Levitzki, Prime ideals and the lower radical, Amer. J. Math. vol. 73 (1951) pp. 25-29.

12. N. H. McCoy, Rings and ideals, Carus Math. Monograph, vol. 8, 1948.

13. - Prime ideals in general rings, Amer. J. Math. vol. 71 (1949) pp. 823-833.

14. M. Nagata, On the theory of radicals in a ring, J. Math. Soc. Japan vol. 3 (1951) pp. 330-344.

15. Y. Sampei, On lattice completions and closure operations, Comm. Math. Univ. St. Paul vol. 2 (1953) pp. 55-70.

Smith College,

NORThAMpton, Mass. 